\title{
A multi-ion beam microanalysis approach for the characterization of plasma polymerized allylamine films
}

\author{
E. Punzón-Quijorna ${ }^{1,2}$, V. Torres-Costa ${ }^{1}$, A. Climent-Font ${ }^{1,2}$, and M. Manso-Silván ${ }^{1, a}$ \\ ${ }^{1}$ Departamento de Física Aplicada, Universidad Autónoma de Madrid, 28049 Madrid, Spain \\ ${ }^{2}$ Centro de Microanálisis de Materiales CMAM, Universidad Autónoma de Madrid, 28049 Madrid, Spain
}

Received: 15 April 2011 / Received in final form: 30 June 2011 / Accepted: 13 July 2011

Published online: 28 October 2011 - (C) EDP Sciences 2011

\begin{abstract}
A full characterization of plasma polymerized biofunctional films requires the use of multianalytical approaches to determine the chemical composition, topography and potential interaction mechanisms of such films with biomolecules and cells. In this work we aim at underlining the versatility of ion-based techniques to contribute to the chemical characterization of plasma polymerized surfaces. The simultaneous use of energy recoil detection (ERD) and Rutherford backscattering (RBS) spectroscopies with incident He ions is an example of this versatility. Performing sequential measurements and the use of correlating computing tools for ERD-RBS interpretation allows providing in-depth concentration profiles of light elements, including namely hydrogen. More accurate analysis of light elements in polymer films can be increased by looking for particular ions with resonant backscattering responses (i.e., non-Rutherford Scattering). In particular, proton beams of $1.765 \mathrm{MeV}$ are used to increase the detection of $\mathrm{C}$ and $\mathrm{N}$, and particular incidence and detector angles to diminish the Si substrate contribution. These analytical tools have been applied to allylamine films and multi-layers crosslinked in a capacitive plasma onto both Si and porous Si substrates.
\end{abstract}

\section{Introduction}

Adapting a material with a specific physical functionality (whether optical, electronic or magnetic) to a biological application requires a modification step to select the desired interaction in its new environment. Plasma polymerization is a reputed method for such process, allowing the chemical activation of diverse material morphologies (from flat homogeneous surfaces [1] to membranes [2,3], hollow structures [4] or beads and particles $[5,6]$ ) by a wide variety of chemical groups (such as amino [6], carboxylic [1] or epoxy [7]). These materials can thus be activated to work in biosensors, enhancing the immobilization of the biomolecule providing the specific recognition of the target species (i.e., enzymes [8], immunoglobulins [9], nucleic acids [10]) or as biomaterials in osteo-articular [3,11], vascular [4] or neural [12] oriented applications.

Optimization of plasma polymerization of monomers bearing polar chemical groups with bioactivation capacity requires a series of characterizations regarding the stability of the layers, the chemistry of the surfaces (namely, the density of functional groups), their topography, the surface charge variations according to the presence of dissociable chemical groups (Zeta potential measurements) and the influence of all these aspects on the final surface free energy. The selection of the plasma generator

\footnotetext{
a e-mail: miguel.manso@uam.es
}

frequency (RF vs. MW), plasma power, polymerization process (i.e., grafting [13] vs. deposition in pulsed [14] or continuous mode $[15,16]$ ) or gas parameters (atmospheric [1] vs. vacuum process, monomer to discharge gas ratio) are key choices determining the final properties of the film.

In the present work we concentrate on the bioactivation of $\mathrm{Si}$ and porous $\mathrm{Si}$ surfaces (PSi) in view of their interest as interface electronics and optoelectronics materials with biological environments. Furthermore, the high specific surface of PSi has promoted this material to be used in different therapeutic approaches [17]. The biofunctionalization is carried out by plasma polymerization of allylamine (All, $\mathrm{CH}_{2}=\mathrm{CH}-\mathrm{CH}_{2}-\mathrm{NH}_{2}$ [18]) in two different conditions: (a) homogeneous plasma conditions during the whole process or (b) power decaying deposition processes (i.e., high initial power to enhance substrate-interface adhesion and final mild conditions compatible with a surface termination with a high surface density of functional groups). We focus on the chemical characterization by ion beam microanalytical techniques (elastic recoil detection (ERD) and Rutherford Backscattering (RBS)). We use not only simultaneous ERD and RBS configurations based on $\mathrm{He}^{+}$as previously used to characterize plasma polymerized films [19], but also non-Rutherford Scattering with $\mathrm{H}^{+}$beams in analog conditions to those defined to analyze hard coatings composed of light elements [20]. 


\section{Experimental}

\subsection{Substrate preparation and PE-CVD}

Deposition of All was performed on Si $\left(\begin{array}{lll}1 & 0 & 0\end{array}\right)$ and PSi substrates. Si ( $\left.\begin{array}{lll}1 & 0 & 0\end{array}\right)$ wafers were cleaned by subsequent acetone and ethanol ultrasound baths and dried in $\mathrm{N}_{2}$ flow. Low resistivity $(0.05-0.1 \Omega \mathrm{cm})$ boron-doped $p$-type Si with $\left(\begin{array}{lll}1 & 0 & 0\end{array}\right)$ orientation was used to grow the PSi layers. The back surface of the Si wafers was coated with $\mathrm{Al}$ by electron beam evaporation and annealed at $400{ }^{\circ} \mathrm{C}$ during $5 \mathrm{~min}$ to grow a low resistance ohmic contact for electrochemical etching. Samples were then galvanostatically etched under illumination (150 W halogen lamp) in a 1:1 HF (49\%):ethanol solution, with a current density of $100 \mathrm{~mA} / \mathrm{cm}^{2}$ for $60 \mathrm{~s}$. The resulting structures were finally rinsed in ethanol and dried under $\mathrm{N}_{2}$ flow resulting in $4 \mu \mathrm{m}$ thick sponge-like PSi.

Deposition of All (Sigma-Aldrich) was performed in a capacitively coupled cylindrical reactor consisting of two $30 \mathrm{~cm}$ radius internal parallel electrodes. Initial chamber background pressure was $5 \times 10^{-2} \mathrm{~Pa}$ (Alcatel Roots Pumping System, Annecy, France) and processing pressure was controlled by a chamber butterfly valve set at $2 \mathrm{~Pa}$. A continuous discharge was applied with a RF source (R.D. Mathis Co. $13.56 \mathrm{MHz}$ ) powered at 50, 100 or $200 \mathrm{~W}$. The substrates were exposed to $20 \mathrm{sccm}$ (equivalent $\mathrm{Ar}$ flow) of All (Fluka) reaching the plasma chamber through a shower inlet during accumulated times of $12 \mathrm{~min}$ (i.e., samples prepared at decaying power were prepared at equal fractions of time accounting for $12 \mathrm{~min}$ ). Samples were labeled according to the precursor (All), the substrate (Si or PSi) and to the plasma power (50, 100, 200 or a sequence of these).

\subsection{Film characterization}

Ion beam microanalytical techniques were used to obtain in-depth elemental information. A CockcroftWalton tandem accelerator located at Centro de MicroAnálisis de Materiales (CMAM, Universidad Autónoma de Madrid, Spain) was used for Rutherford backscattering spectroscopy (RBS) and elastic recoil detection analysis (ERD). RBS and ERD measurements on All/PSi samples were performed simultaneously with a $2 \mathrm{MeV} \mathrm{He}^{+}$beam at an incidence angle of $75^{\circ}$ with respect to the surface normal. The RBS and ERD spectra were acquired by using two silicon surface barrier detectors placed at scattering angles of $170^{\circ}$ and $30^{\circ}$, respectively. A $13 \mu \mathrm{m}$ thick mylar foil was placed in front of the ERD detector to filter the He scattered particles from the $\mathrm{H}$ recoils. Three consecutive spectra were obtained $(3 \mu \mathrm{C} /$ shot $)$ at the same position of every All surface in order to evaluate the damage induced by successive exposure to the probing beam. All spectra were simulated using the SIMNRA code [21] to obtain the element in-depth composition.

RBS and non-RBS measurements were performed on All/Si surfaces with a $1.765 \mathrm{MeV} \mathrm{H}^{+}$beam. Two angular configurations for the detector were studied:

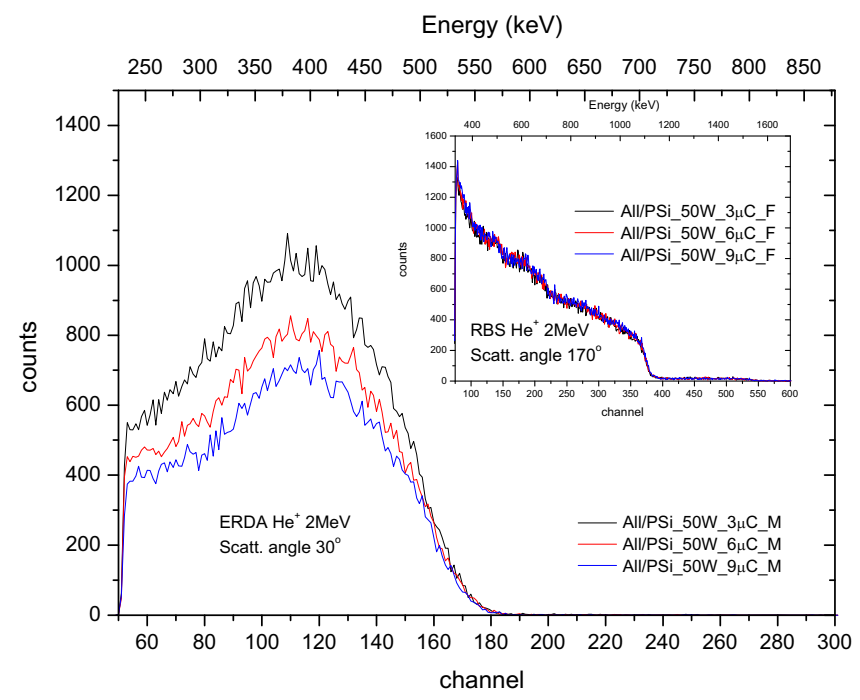

Fig. 1. (Color online) Ion beam damage on All/PSi_50 film after three consecutive ERD and RBS (inset) simultaneous analysis with $2 \mathrm{MeV} \mathrm{He}^{+}$ions $(3 \mu \mathrm{C} /$ shot $)$.

(a) detection at a scattering angle of $170^{\circ}$ to enhance the non-Rutherford resonance of C12 at this energy and (b) detection at a scattering angle of $128^{\circ}$ to reduce the Si background signal and improve $\mathrm{C}$ and $\mathrm{N}$ detection.

\section{Results and discussion}

The use of ion beam microanalysis techniques for the characterization of soft matter samples implies taking into account the potential damage induced in the sample by the impinging ions [22]. Damage to All/PSi samples was studied by carrying out cumulative shot experiments placing the detectors for simultaneous ERD and RBS acquisition. Figure 1 shows the ERD spectra corresponding to All/PSi_50 samples at the different shots. It is patent by the figure that the ion beam irradiation induces a depletion of $\mathrm{H}$ content in the films. The $\mathrm{H}$ losses are not equally distributed in depth (surface composition on the top of the surface seems to be relatively independent of the shot number while the differences are patent in deeper regions) as derived from the absence of a proportionality factor that could eventually make all these spectra match. Nevertheless, the observation of the simultaneous RBS spectra at identical point illustrates that there is no drastic modification of the polymerized backbone at the accumulated charges used (Fig. 1, inset).

Both ERD and RBS spectra obtained from All/PSi_50 and All/PSi_200 were simultaneously fitted by using the SIMRA code as shown in Figures $2 \mathrm{a}$ and $2 \mathrm{~b}$ for ERD and RBS, respectively. Fitting of the three consecutive ERD spectra allowed extrapolating to the original $\mathrm{H}$ content at zero ion irradiation. RBS confirmed that $200 \mathrm{~W}$ deposition allowed higher polymer fragment retention thus producing a thicker film (see retarded Si signal counts for this sample). Furthermore, RBS spectra presented additional contributions from three light elements $(\mathrm{C}, \mathrm{N}, \mathrm{O})$ and a trace signal from $\mathrm{Cu}$ (PSi electrochemical etch contaminant). 
E. Punzón-Quijorna et al.: Ion beam microanalysis of plasma polymerized allylamine films
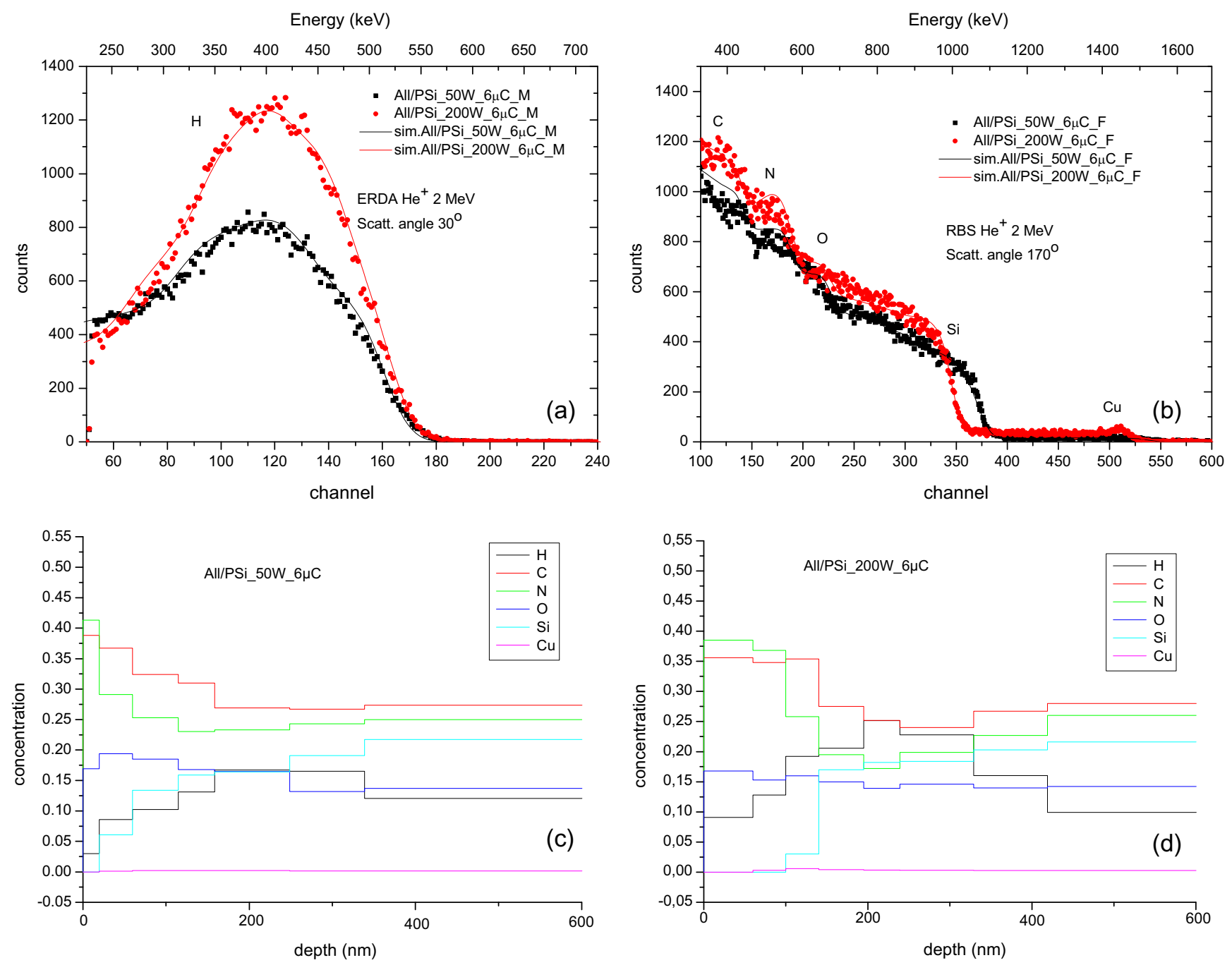

Fig. 2. (Color online) Simultaneous ERD and RBS analysis of All/PSi_50 and All/PSi_200 with $2 \mathrm{MeV}^{\mathrm{H}} \mathrm{H}^{+}$: (a) ERD experimental and simulated spectra, (b) RBS experimental and simulated spectra. Derived in-depth profiles for (c) All/PSi_50 and (d) All/PSi_200.

The simulation of the RBS spectra allowed obtaining indepth concentration profiles, as illustrated in Figures 2c and 2d for All/PSi_50 and All/PSi_200, respectively. For the All/PSi_50 sample the polymerized film is extremely thin with detection of the PSi constituents (both Si and O) at a depth of 10-20 nm. At this surface layer the composition of $\mathrm{N}$ is higher than $40 \%$. By going into deeper layers, the composition seems to vary smoothly allowing the detection of $\mathrm{C}$ and $\mathrm{N}$ at depths higher than $100 \mathrm{~nm}$. With regard to the All/PSi_200 sample, it is evidenced that a film of approximately $140 \mathrm{~nm}$ grows onto the PSi substrate. However, this film seems to penetrate less into the PSi and presents a more abrupt profile. It is also relevant that the $\mathrm{N}$ composition on the top surface is approximately $5 \%$ below what obtained for All/PSi_50 films. These aspects reveal that, the permeability of PSi to the activated species is rather limited by an increase in the reactivity of All monomer fragments than by the size of these fragments. It is patent in fact that the smaller and more reactive fragments obtained at $200 \mathrm{~W}$ saturate the pores and block the polymer film ingrowth.
The previous analysis shows that the use of $\mathrm{He}^{+}$beams induces very low signal counts for light elements in the RBS spectra. In order to enhance the signal, a proton beam was used to analyze the All films deposited on flat Si substrates. Figure 3a shows the non-Rutherford Scattering spectra obtained at $170^{\circ}$ scattering angle corresponding to All films prepared at increasing plasma powers. It can be observed that the sensibility toward $\mathrm{C}$ has dramatically increased. Such configuration is much more sensible to the $\mathrm{C}$ integration and illustrates how the backbone retention increases for increasing power in the range of deposition of 50-200 W. However, this angular configuration extremely enhances the Si substrate signal hiding the potential contributions from $\mathrm{N}$ and $\mathrm{O}$. In such configuration the simulation of the spectra leads to film compositions considerably different from what obtained in the previous example analyzed with $\mathrm{He}^{+}$. Figure $3 \mathrm{~b}$ shows the in-depth composition profile obtained for the All/Si_200 film. In fact, the profile shows that film thickness obtained is similar to what measured on top of All/PSi_200. The profile reproduces quite well the $\mathrm{Si}$ substrate composition with the $\mathrm{SiO}_{x}$ 
The European Physical Journal Applied Physics
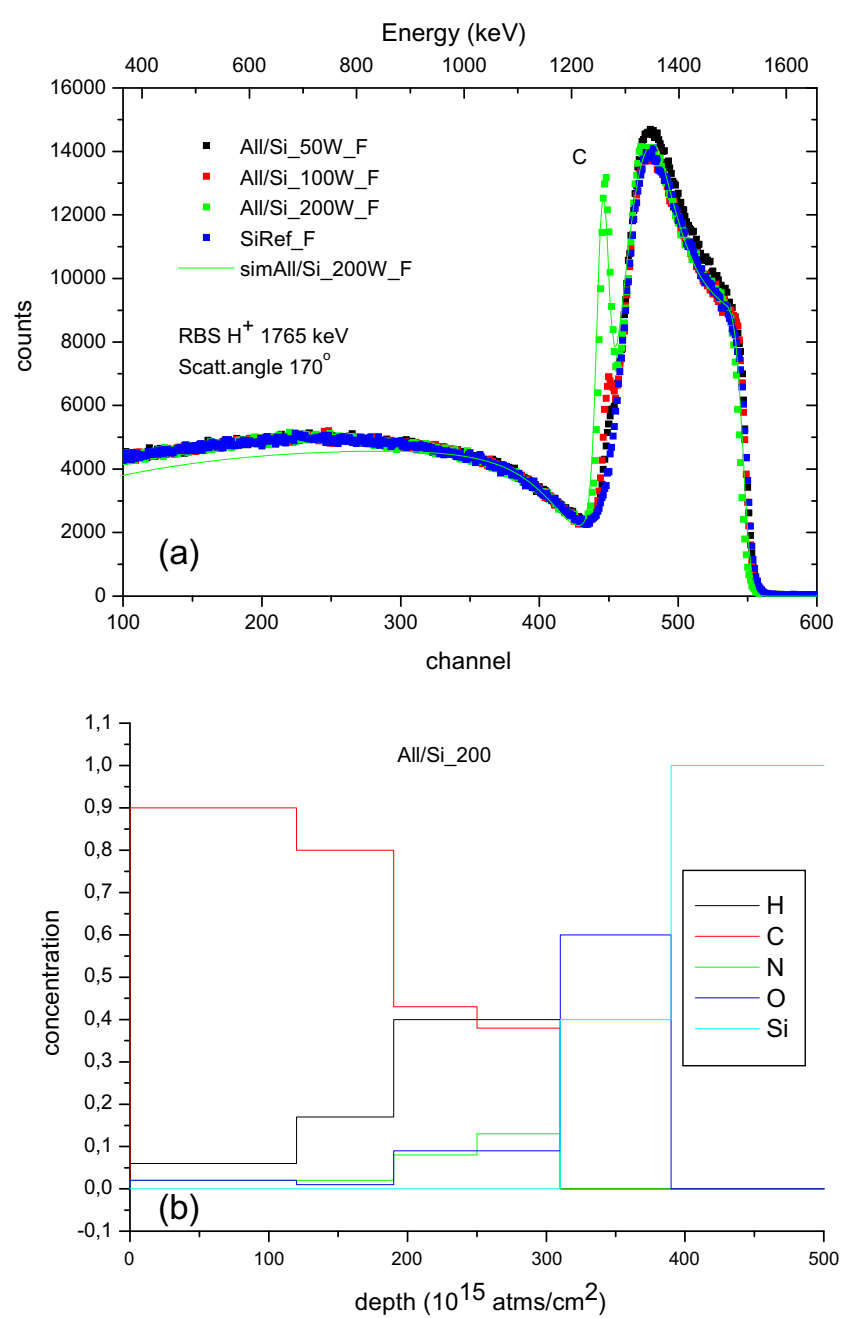

Fig. 3. (Color online) RBS analysis of All/Si_50, All/Si_100 and All/Si_200 with $1.765 \mathrm{MeV} \mathrm{H}^{+}$: (a) experimental spectra at $170^{\circ}$ scattering angle and illustrative simulated spectrum for All/Si_200, (b) derived in-depth profiles for All/PSi_200.

passivation layer. However, the composition of $\mathrm{N}$ is remarkably below the levels indicated in the equivalent All/PSi spectra.

The All films on Si substrates were optimized in terms of stability and surface composition by gradually decreasing the plasma power during polymerization. Figure $4 \mathrm{a}$ shows the characteristic non-Rutherford Scattering spectra of gradual films with respect to those deposited homogeneously at $200 \mathrm{~W}$ (all $170^{\circ}$ scattering angle). The spectra agree with the idea that several minutes of $200 \mathrm{~W}$ deposition (6 min for 200_50 sequence and 4 min for 200_100_50 sequence) allow integrating a crosslinked polymer film at the interface. However, such configuration allowed again only poor detection of integrated N. In these circumstances we explored a new angular configuration that could improve $\mathrm{N}$ signal. Figure $4 \mathrm{~b}$ shows the characteristic nonRutherford Scattering spectra of the same series of gradual films after shifting the scattering angle to $128^{\circ}$. The advantage of such configuration can be summarized in two features: (a) There is a considerable recovery of in-depth
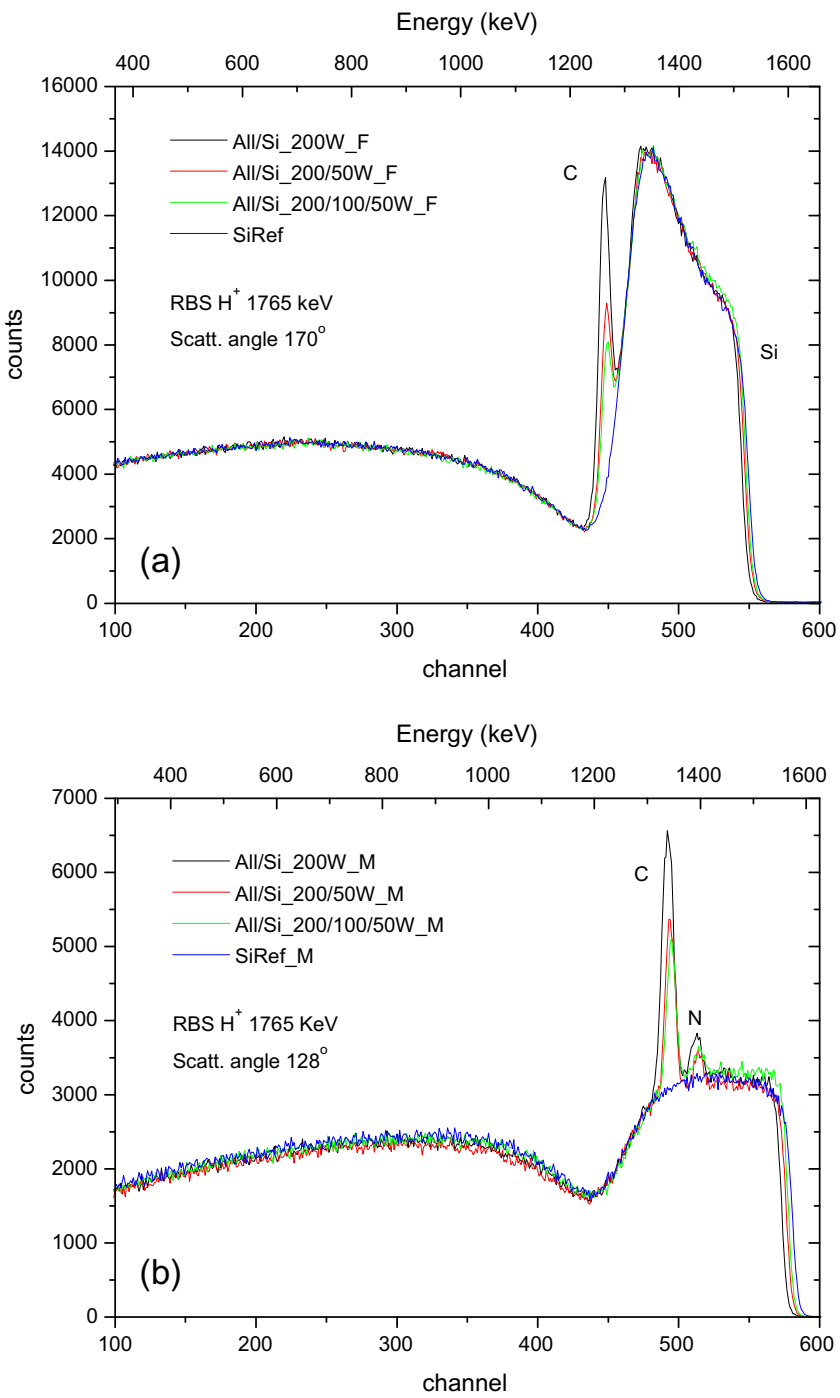

Fig. 4. (Color online) RBS analysis of All/Si layers grown in gradual plasma power softening conditions measured with $1.765 \mathrm{MeV} \mathrm{H}^{+}$: (a) experimental spectra at $170^{\circ}$ scattering angle, (b) experimental spectra at $128^{\circ}$ scattering angle.

resolution allowing to clearly state the following decreasing order in fragment integration within the All film: All/ Si_200 > All/Si_200_100 > All/Si_200_100_50 (see the energy attributed to the raising Si substrate signal). (b) The $\mathrm{N}$ signal now clearly emerges from the Si background allowing more accurate estimations of $\mathrm{N}$ composition. This latter advantage allows observing that by reducing the plasma polymerization power, the relative composition of $\mathrm{N}$ with respect to $\mathrm{C}$ can be increased, thus supporting the idea of an increased capacity for amine group retention on the top surface. Techniques for chemical shift capacity determination are to be used to determine if the detected $\mathrm{N}$ is actually in biofunctional primary amine state [16].

\section{Conclusions}

Ion beam microanalysis has been successfully used to characterize plasma polymerized films. We show that care 
should be taken to account for the damage induced by the impinging ions. For All films deposited on PSi, a conventional configuration of simultaneous ERD and RBS measurements with $2 \mathrm{MeV} \mathrm{He}{ }^{+}$beam gives a satisfactory in-depth and elemental resolution. This was used to describe features relative to permeability of active plasma fragments into the pores. It is concluded that penetration of fragments is limited rather by increasing reactivity than by the size of the fragments. For the analysis of All films on flat $\mathrm{Si}$, the detection capacity of $\mathrm{C}$ was increased by working in non-Rutherford conditions $(1.765 \mathrm{MeV}$ with detector at $170^{\circ}$ scattering angle). In such configuration $\mathrm{N}$ detection is drastically reduced, but a change of location of the detector to a scattering angle of $128^{\circ}$ allows accounting for $\mathrm{N}$ composition. In such configuration it has been possible to confirm that, by gradual deposition, a significant film retention can be obtained by starting deposition process at $200 \mathrm{~W}$. Such retention is compatible with an increased surface $\mathrm{N}$ content by finishing deposition at $50 \mathrm{~W}$. All these analyses could be compared with results from alternative microanalysis configurations, including the use of ERD with higher mass ions such as $\mathrm{Si}^{+}$or $\mathrm{Cl}^{+}$, and should be complemented with techniques providing information on topographic, chemical state and possible charge or dipole interactions of the films in biomolecular or cellular systems.

We are especially thankful to L. García Pelayo for his assistance during materials processing and characterization. We acknowledge MICINN funding provided by Grant No. MAT2008-06858-C02-01 and grant from Fundación Domingo Martínez.

\section{References}

1. M. Tatoulian, F. Arefi-Khonsari, J.P. Borra, Plasma Process. Polym. 4, 360 (2007)

2. J.R. Hollahan, T. Wydeven, Science 179, 500 (1973)

3. F. Formosa, V. Sanchez-Vaquero, C. Rodriguez-Navas, A. Munoz-Noval, N. Tejera-Sanchez, M.M. Silvan, J.P. Garcia-Ruiz, G. Marletta, Plasma Process. Polym. 7, $794(2010)$
4. S.J. Song, K.S. Kim, K.H. Kim, H.J. Li, J.H. Kim, M.H. Jeong, B.H. Kim, Y.M. Ko, D.L. Cho, J. Mater. Chem. 19, 3248 (2009)

5. J.C. Shearer, M.J. Fisher, D. Hoogeland, E.R. Fisher, Appl. Surf. Sci. 256, 2081 (2010)

6. M. Manso, A. Rodriguez, A. Paul, J. Barrero, F. Rossi, Surf. Interface Anal. 38, 322 (2006)

7. B. Thierry, M. Jasieniak, L. de Smet, K. Vasilev, H.J. Griesser, Langmuir 24, 10187 (2008)

8. Y. Matsui, M. Yoshizawa, T. Hoshino, H. Muguruma, Sens. Mater. 20, 289 (2008)

9. S. Kurosawa, N. Kamo, T. Arimura, A. Sekiya, M. Muratsugu, Jpn. J. Appl. Phys. 34, 3925 (1995)

10. K.S. Chen, S.C. Chen, H.R. Lin, T.R. Yan, C.C. Tseng, Mater. Sci. Eng. C 27, 716 (2007)

11. B. Finke, F. Luethen, K. Schroeder, P.D. Mueller, C. Bergeniann, M. Frant, A. Ohl, B.J. Nebe, Biomaterials 28, 4521 (2007)

12. M. Buttiglione, F. Vitiello, E. Sardella, L. Petrone, M. Nardulli, P. Favia, R. d'Agostino, R. Gristina, Biomaterials 28, 2932 (2007)

13. J.R. Ren, J. Wang, H. Sun, N. Huang, Appl. Surf. Sci. 255, 263 (2008)

14. G. Mishra, S.L. McArthur, Langmuir 26, 9645 (2010)

15. J. Behnisch, J. Tyczkowski, M. Gazicki, I. Pela, A. Hollander, R. Ledzion, Surf. Coat. Technol. 98, 872 (1998)

16. M.M. Silvan, A. Valsesia, M. Hasiwa, D. Gilliland, G. Ceccone, F. Rossi, Chem. Vapor Depos. 13, 211 (2007)

17. R.J. Martin-Palma, M. Manso-Silvan, V. Torres-Costa, J. Nanophotonics 4, 042502 (2010)

18. P. Hamerli, T. Weigel, T. Groth, D. Paul, Surf. Coat. Technol. 174, 574 (2003)

19. S.F. Yoon, X. Rusli, J. Ahn, Q. Zhang, C.Y. Yang, H. Yang, F. Watt, Thin Solid Films 340, 62 (1999)

20. A. Climent-Font, M. Cervera, M.J. Hernandez, A. Munoz-Martin, J. Piqueras, Nucl. Instr. Methods B 266, 1498 (2008)

21. M. Mayer, in Proc. of 15th Int. Conf. on the Application of Accelerators in Research and Industry, vol. 475, edited by J.L. Duggan, I.L. Morgan (AIP Press, New York, 1999), p. 541

22. M.M. Silvan, R. Gago, A. Valsesia, A.C. Font, J.M.M. Duart, F. Rossi, Nucl. Instr. Methods B 257, 496 (2007) 